\title{
UNDERSTANDING THE ORBITAL TRANSFER VEHICLE TRADE SPACE
}

\author{
Hugh L. McManus* \\ Metis Design, 46 Second St., Cambridge, MA 02140 \\ and Todd E. Schuman ${ }^{\dagger}$ \\ Massachusetts Institute of Technology, Cambridge MA 02139
}

\begin{abstract}
This study uses new methods to explore the theoretical performance of over a hundred possible orbital transfer vehicle designs. The designs have varying propulsion types, fuel mass fractions, and grappling/observation equipment capabilities. Simple sizing rules are used to calculate the performance of the designs and their utility to several types of users. Designs of interest are further explored using Integrated Concurrent Engineering techniques, resulting in complete conceptual designs. The results give an understanding of the trade-space for such vehicles, including sensitivities to both design variables and assumed user needs. This clarifies some of the challenges involved such as physical constraints and sensitivities to uncertain user preferences. Several potentially viable designs are identified including an electric-propulsion high delta-V vehicle dubbed the Electric Cruiser, and a class of lower delta- $\mathrm{V}$ vehicles dubbed Tenders which are studied in a companion paper.
\end{abstract}

\section{NOMENCLATURE}

$\begin{array}{ll}C & \text { Cost (\$) } \\ c_{d} & \text { Dry mass cost coefficient }(\$ / \mathrm{kg}) \\ c_{w} & \text { Wet mass cost coefficient }(\$ / \mathrm{kg}) \\ I_{s p} & \text { Specific impulse }(\mathrm{sec}) \\ g & \text { Acceleration due to gravity }\left(9.8 \mathrm{~m} / \mathrm{sec}^{2}\right) \\ M_{b} & \text { Bus mass }(\mathrm{kg}) \\ m_{b f} & \text { Bus mass fraction coefficient } \\ M_{c} & \text { Mass of observation/manipulator system }(\mathrm{kg}) \\ M_{d} & \text { Dry mass }(\mathrm{kg}) \\ M_{f} & \text { Fuel mass }(\mathrm{kg}) \\ M_{p} & \text { Mass of propulsion system }(\mathrm{kg}) \\ m_{p 0} & \text { Propulsion system base mass }(\mathrm{kg}) \\ m_{p f} & \text { Propulsion system mass fraction coefficient } \\ M_{w} & \text { Wet mass (kg) } \\ U_{t o t} & \text { Total utility } \\ V_{c} & \text { Single attribute utility for capability } \\ V_{t} & \text { Single attribute utility for response time } \\ V_{v} & \text { Single attribute utility for delta-V } \\ W_{c} & \text { Utility weighting for capability } \\ W_{t} & \text { Utility weighting for response time } \\ W_{v} & \text { Utility weighting for delta-V } \\ \square v & \text { Change in velocity (m/sec) }\end{array}$

\section{INTRODUCTION}

An orbital transfer vehicle, or "space tug," is one instance of a broad class of vehicles that can perform a variety of on-orbit servicing functions. The simplest function of such a vehicle would be to observe space assets, hereafter referred to as targets, in situ. The targets may be cooperative (designed for servicing), partially cooperative (e.g. maneuverable in ways helpful to the tug), uncooperative (inert), or even hostile. The later case covers spinning or tumbling vehicles that would be hazardous to approach. A tug changes the orbits of these targets for operational reasons (e.g. life extension), to retrieve the targets, bringing them out of orbit or to other assets (e.g. Shuttle or ISS), or to eliminate debris. Similar vehicles may interact or service targets in a variety of other ways. The ability to interact with objects in space is a desirable capability, but clearly the range of possible approaches is large, and it has proven difficult to design viable tug systems.

The concept of tug vehicles goes back to the early years of the space program. A literature review is included in a companion paper. ${ }^{1}$ Here, we will only note that hypothetical tugs designed for a single mission rarely show an economic pay-off, although there is some evidence that if an infrastructure for on-orbit service could be created it would have positive value. ${ }^{2}$ The concept in practice is made difficult by unfriendly orbital dynamics (many desired maneuvers are extremely energy-intensive), environments (the vehicle must be radiation hard, and/or hard against some level of debris damage, to last useful lifetimes in many orbits), and economics (markets are uncertain, and payoff is difficult to prove). Some missions require nuclear or other advanced propulsion systems, and most require advances in control systems and docking or grappling hardware.

In this work, new space system architecture and conceptual design techniques have been applied to the tug problem. A capability referred to as Multi-Attribute Tradespace Exploration (MATE) with Concurrent Engineering (MATE-CON) was used. MATE is a method for examining many design concepts to

\footnotetext{
* Senior Special Projects Engineer, Associate Fellow AIAA

'Graduate Research Assistant, Department of Aeronautics and Astronautics

Copyright (C) 2003 by Hugh L. McManus. Published by the American Institute of Aeronautics and Astronautics, Inc. with permission.
} 
understand the possibilities and problems of the space of possible solutions - the tradespace. ${ }^{3}$ It was developed at MIT from earlier work on information systems analysis applied to space systems. ${ }^{4}$ Integrated Concurrent Engineering (the CON in MATE-CON, but usually referred to on its own as ICE) is a method for rapidly producing preliminary designs in a "design room" environment. The system used in this study descends from work at $\mathrm{JPL}^{5}$ and the Aerospace Corporation, ${ }^{6}$ by way of Caltech. ${ }^{7}$ The overall MATECON system, along with other front-end design tools, was developed by a consortium of MIT, Caltech, and Stanford. ${ }^{8}$

Using MATE, several hundred possible space tug vehicles are evaluated for their ability to move mass in orbit and interact with targets. The resulting tradespace is examined to clarify some of the fundamental difficulties with the space tug concept, understand the sensitivities of the tradespace to uncertainties in users needs, identify the Pareto front of "good" designs, and find some design points that are promising for multipurpose tugs. ICE is then used to create ten conceptual designs for a range of hypothetical mission scenarios. The ICE designs lend credibility to the crude MATE models, further clarify design issues, and provide a starting point for further development of missions of interest.

This paper covers the MATE and ICE models created to do the analyses, the MATE tradespace and its interpretation, and the conceptual design of four tug vehicles for a mission involving the rescue of a Geosynchronous Earth Orbit (GEO) satellite stranded in a transfer orbit by the failure of its apogee motor. A companion paper looks at a variety of specific missions, suggested originally by this tradespace analysis, that concentrate on servicing groups of satellites in similar orbits.

\section{MATE METHOD}

In MATE, user needs are defined in terms of the system's attributes, or capabilities of the desired system, rather than the characteristics of the desired space vehicle. These needs are expressed and quantified in utility metrics, often through the use of Multi-Attribute Utility Theory. Then a design vector is selected, consisting of a very large number (hundreds to hundreds of thousands) of possible systems that could be used to meet the user needs. Simulation models are used to calculate the attributes of the proposed systems. The systems are then evaluated against the users' utilities to understand which systems best satisfy the users' needs. The results, collectively referred to as the tradespace, can then be explored. This process consists of the search for not only optimal solutions, but also for understanding of design sensitivities, key trade-offs, dangerous uncertainties, and vulnerabilities to changes in the market or national policy. Often these understandings will change a user's perception of his or her need, and/or the designer's perception of the appropriate design space, resulting in a need to repeat the analysis. The semi-automated nature of the computations allows this valuable exploitation of emergent understanding with little cost or time penalty. Eventually, a design or designs from the trade space are selected for further consideration.

In this study, a somewhat simplified version of the MATE method was used. The method was adapted in response to difficulties including the lack of an immediate customer and a very open design space. The customer utilities were handled parametrically to understand the sensitivities of the tradespace to ranges of, and changes in, user needs. The analysis was done at a high level, using low-fidelity models, but covering a large range of possible designs.

\section{Attributes and Utilities}

The capabilities of a space tug vehicle determined to be useful to a potential user include: (1) total delta-V capability, which determines where the spacetug can go and how far it can change the orbits of target vehicles; (2) mass of observation and manipulation equipment (and possibly spare parts, etc.) carried, which determines at a high level what it can do to interact with targets, referred to here as its capability; and (3) response time, or how fast it can get to a potential target and interact with it in the desired way. Note that the design of observation and manipulation equipment and its corresponding software is outside the scope of this study - the equipment is treated as a "black box" with mass and power requirements.

These attributes are translated into a single utility function. In the absence of real users from which to collect more sophisticated functions, ${ }^{9}$ it was decided that a simple function that could be explored parametrically was most appropriate. The three attributes are assigned single-attribute utilities. These are dimensionless metrics of user satisfaction from zero (minimal user need satisfied) to one (fully satisfied user). The utilities are combined as a weighted sum.

The delta-V utility is shown in Fig. 1. Delta-V is a continuous attribute calculated for each system considered. Utility is assumed to increase linearly with delta-V, with diminishing returns above the levels necessary to do Low Earth Orbit (LEO) to GEO transfers. Variations on this utility are shown in Figs. 2 and 3, which show respectively the utilities of a GEOcentric user (large steps in utility for achieving GEO and GEO round-trip capabilities) and a delta-V-hungry user (continued linear utility for very high delta-V). The manipulator mass (capability) attribute has discrete values, assumed to correspond to increasing utility as shown in Table 1 . The response time of a real system would be a complex function of many factors; at the level of the current analysis it is reduced to a binary attribute $V_{t}$, valued at one for high impulse systems, and zero for low impulse ones. 
The combined utility is calculated as follows:

$$
U_{\text {tot }}=W_{v} V_{v}+W_{c} V_{c}+W_{t} V_{t}
$$

The combined utility is a dimensionless ranking of the presumed usefulness of the system to a nominal user. It needs to be interpreted with care, as it provides ranking ( 0.8 is better than 0.4 ) but not scale ( 0.8 is not

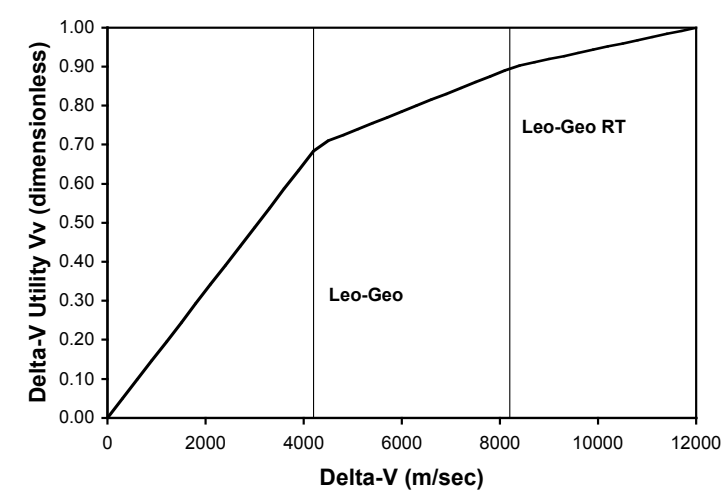

Fig. 1. Nominal single attribute utility for delta-V

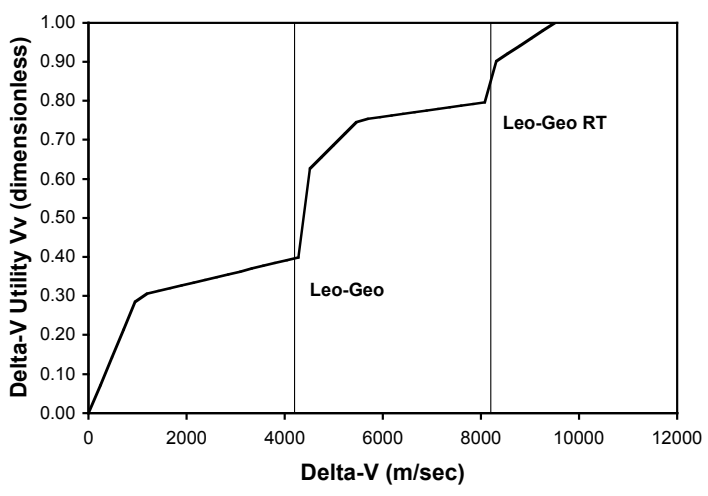

Fig. 2. Delta-V utility for GEO-centric user

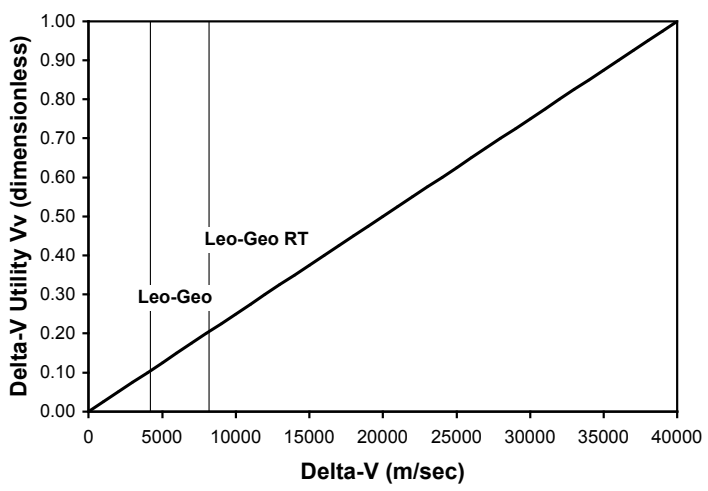

Fig. 3. Delta-V utility for delta-V hungry user necessarily twice as good as 0.4 ) or any physical meaning. The nominal weightings and two other cases studied are shown in Table 2.

Design vector and calculation of attributes

A set of design variables (in MATE parlance, a design vector) was selected to represent possible tug vehicles. The following variables were selected: (1) observation and manipulator system mass; (2) propulsion type, and (3) mass of fuel carried.

Table 1 shows the relationship assumed between manipulator mass, assumed capability, and utility value. No attempt was made to design or even specify the manipulator system, but for reference the $300 \mathrm{~kg}$ size is typical of small industrial robots, while the high capability $(3000 \mathrm{~kg})$ is taken from a postulated system based on shuttle arm technology.

Table 3 shows the choices of propulsion system considered, along with some assumed properties of the propulsion systems. The total mass of the propulsion system is taken to be

$$
M_{p}=m_{p 0}+m_{p f} M_{f}
$$

The fuel mass $M_{f}$ was set at 30, 100, 300, 600, 1200, $3000,10000,30000$ or $50000 \mathrm{~kg}$, obviously spanning a large range of possible delta-Vs.

Table 1. Manipulator capability attribute, with corresponding utility and mass

\begin{tabular}{lcc}
\hline \hline Capability & $\begin{array}{c}\text { Utility value } V_{c} \\
\text { (dimensionless) }\end{array}$ & $\begin{array}{c}\text { Mass } M_{c} \\
(\mathrm{~kg})\end{array}$ \\
\hline Low & 0.3 & 300 \\
Medium & 0.6 & 1000 \\
High & 0.9 & 3000 \\
Extreme & 1.0 & 5000 \\
\hline \hline
\end{tabular}

Table 2. Utility weightings

\begin{tabular}{lccc}
\hline \hline Attribute & $\begin{array}{c}\text { Nominal } \\
\text { Weights }\end{array}$ & $\begin{array}{c}\text { Capability } \\
\text { Stressed }\end{array}$ & $\begin{array}{c}\text { Response } \\
\text { Time } \\
\text { Stressed }\end{array}$ \\
\hline Delta-V & 0.6 & 0.3 & 0.2 \\
Capability & 0.3 & 0.6 & 0.2 \\
Response Time & 0.1 & 0.1 & 0.6 \\
\hline \hline
\end{tabular}

Table 3. Propulsion system choices and characteristics

\begin{tabular}{lrrrc}
\hline \hline $\begin{array}{l}\text { Propulsion } \\
\text { System }\end{array}$ & $\begin{array}{c}I_{s p} \\
(\mathrm{sec})\end{array}$ & $\begin{array}{c}\text { Base } \\
\text { Mass } \\
m_{p 0}(\mathrm{~kg})\end{array}$ & $\begin{array}{c}\text { Mass } \\
\text { Fract. } \\
m_{p f}\end{array}$ & $\begin{array}{c}\text { High } \\
\text { Impulse }\end{array}$ \\
\hline Storable biprop & 300 & 0 & 0.12 & $\mathrm{Y}$ \\
Cryo & 450 & 0 & 0.13 & $\mathrm{Y}$ \\
Electric & 3000 & 25 & 0.30 & $\mathrm{~N}$ \\
Nuclear & 1500 & 1000 & 0.20 & $\mathrm{Y}$ \\
\hline \hline
\end{tabular}


The design vector described above represents 144 possible designs. A few of the more extreme of these designs were omitted, more for clarity of the resulting graphics than for computational ease. A few designs with intermediate values of fuel mass, corresponding to specific missions described in this and the companion paper, were added; the final design vector contained 137 possible designs.

The attributes of each design were calculated as follows. The capability, and its utility, are determined directly from the manipulator system mass as shown in Table 1. The response time attribute is determined directly from the propulsion system choice. Those capable of high impulse are given a response time utility $V_{t}$ of one; those not capable are given a $V_{t}$ of zero. The delta-V attribute, and the cost, are calculated by some simple vehicle sizing rules and the rocket equation.

The vehicle bus mass is calculated as

$$
M_{b}=M_{p}+m_{b f} M_{c}
$$

The vehicle dry mass is calculated as

$M_{d}=M_{b}+M_{c}$

and the vehicle wet mass is

$$
M_{w}=M_{d}+M_{f}
$$

The total delta-V attribute is then

$$
\square v=g I_{s p} \ln \left(M_{w} / M_{d}\right)
$$

The delta-V utility is then calculated (by an interpolation routine) from Fig. 1, Fig. 2, or Fig. 3. Note that Eq. 6 calculates the total delta-V that the vehicle can effect on itself. Use of this value is supported by the fact that most missions studied spend most of their fuel maneuvering the tug vehicle without an attached target. Alternately, this delta- $\mathrm{V}$ can be thought of as a commodity. If a target vehicle is attached to the tug, more of this commodity must be expended. Mission specific true delta-V's for a variety of missions are discussed in the companion paper.

The individual utilities having been calculated, the total utility is calculated using Eq. 1. The first-unit delivered cost is estimated based on a simple rule-of-thumb formula.

$$
C=c_{w} M_{w}+c_{d} M_{d}
$$

Equation 7 accounts for launch and first-unit hardware procurement costs. Technology development costs are not included. The values for the coefficients in Eqs. 2,
3, 6, and 7 are found in Tables 3 and 4. These values comprise the constants vector in MATE parlance. The calculations are set up so that these values can be easily altered. These values were varied $+/-10 \%$ and no strong sensitivity was found to any of them. However, it must be noted that some of them (e.g. the nuclear propulsion properties) are quite speculative, and the trade space may look different if they were drastically altered.

Table 4. Misc. Coefficients

\begin{tabular}{cc}
\hline \hline Constant & Value (units) \\
\hline$m_{b f}$ & $1($ dimensionless) \\
$c_{w}$ & $20(\mathrm{k} \$ / \mathrm{kg})$ \\
$c_{d}$ & $150(\mathrm{k} \$ / \mathrm{kg})$ \\
\hline \hline
\end{tabular}

\section{MATE RESULTS}

Figure 4 shows the tradespace as a plot of utility vs. cost with each point representing an evaluated design. The Pareto front of desirable designs are down (low cost) and to the right (high performance). The Pareto front features an area of low-cost, lower utility designs (at the bottom of Fig. 4). In this region, a large number of designs are available, and additional utility can be had with moderate increase in cost. On the other hand, very high levels of utility can only be purchased at great cost (right hand side of plot).

The propulsion system is highlighted in Fig. 4, with different symbols showing designs with different propulsion systems. The propulsion system is not a discriminator in the low-cost, low utility part of the Pareto front, except that nuclear power is excluded. At the high end, on the other hand, the Pareto front is populated by nuclear-powered designs. Electric propulsion occupies the "knee" region where high utility may be obtained at moderate cost

Figure 5 shows the cost banding due to different choices of manipulator mass, or capability. For the lower-performance systems, increased capability translates to large increases in cost with only modest increases in utility. High capabilities are only on the Pareto front for high utility, very high cost systems. This indicates, for the nominal set of user utilities used, cost effective solutions would minimize the mass and power of the observation and manipulation systems carried. Using the utility weights for the "Capability Stressed" user (Table 2) results in Fig. 6. As expected, increasing capability systems now appear all along the Pareto front, although capability still comes at a fairly steep price. 


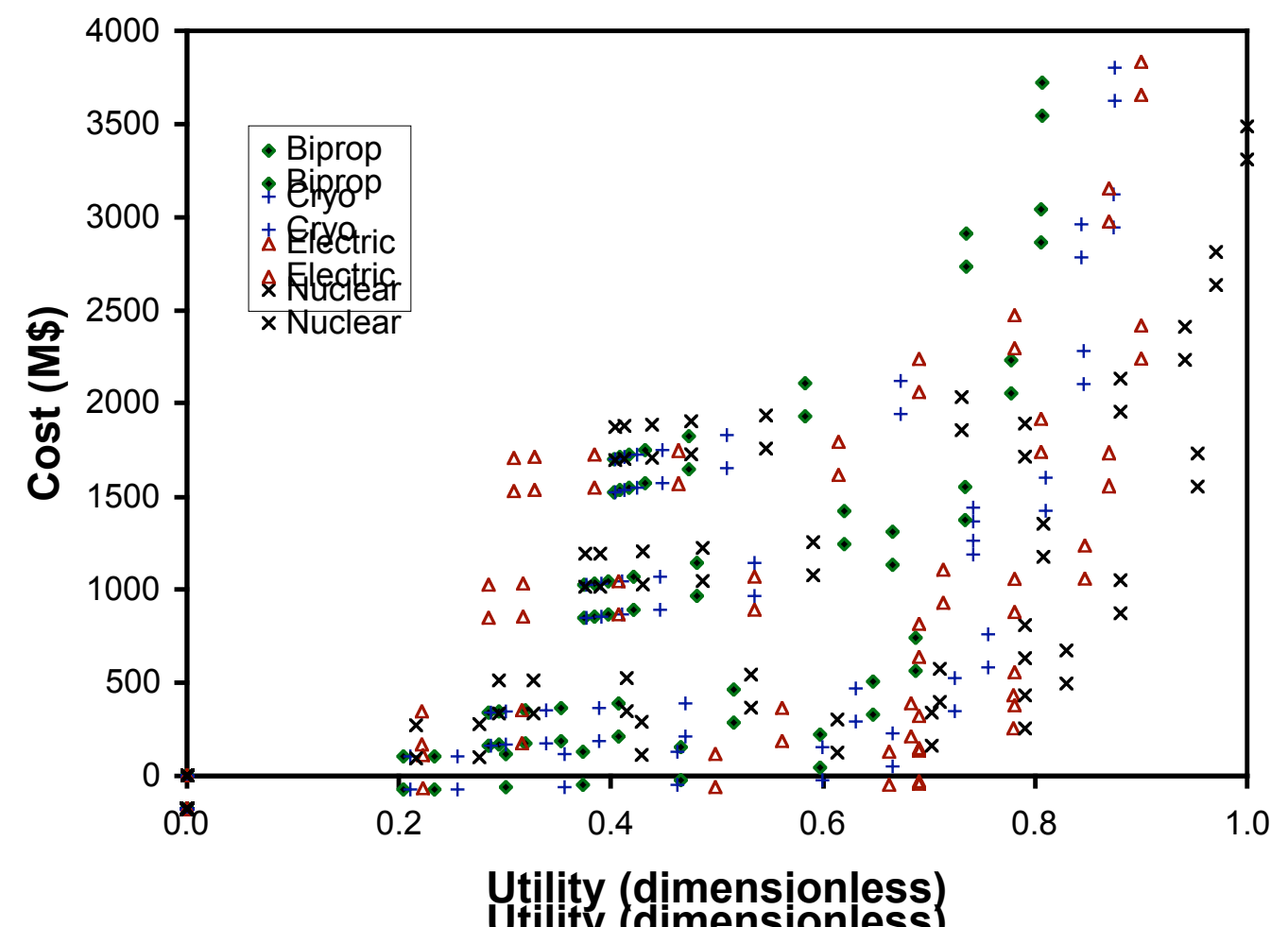

Fig. 4. Trade space for nominal user, with propulsion system indicated

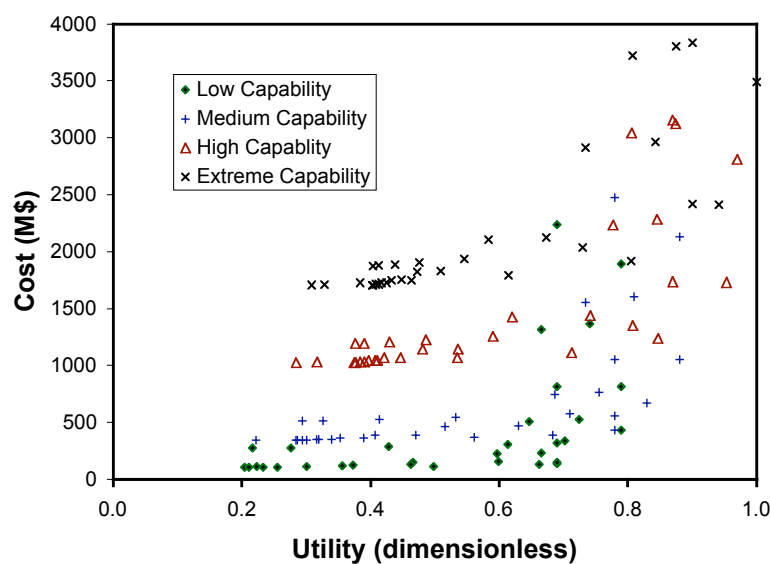

Fig. 5. Trade space for nominal user, with capability indicated

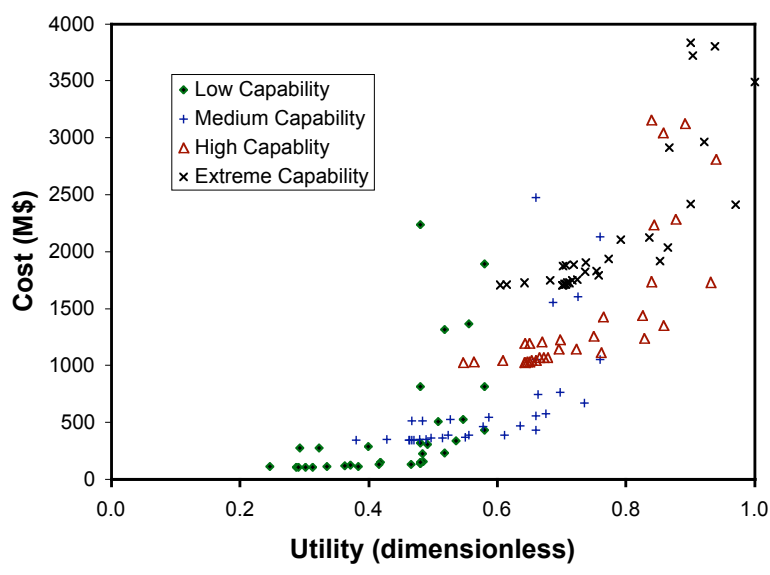

Fig. 6. Trade space for capability stressed user

5

American Institute of Aeronautics and Astronautics 
Using the utility weightings for the "Response Time Stressed" user (Table 2) results in Fig. 7. The results are clear; electric propulsion is eliminated from consideration. In the nominal case (Fig. 4) electric propulsion appears at the "knee" of the Pareto front, and would appear to give good utility for modest cost, but that conclusion will be very sensitive to the weighting given response time by an actual user. Conversely, if the nominal weights and the delta-V utility function from Fig. 3 are used (representing a user with a demand for very large delta-V) the result is Fig. 8 . Now, almost all the designs on the Pareto front feature electric propulsion.

A more detailed view of the lower right-hand corner of the nominal Pareto front (from Fig. 4) is shown in Fig. 9. Only low-capability systems are shown. The lines connect designs that differ only by fuel load carried.

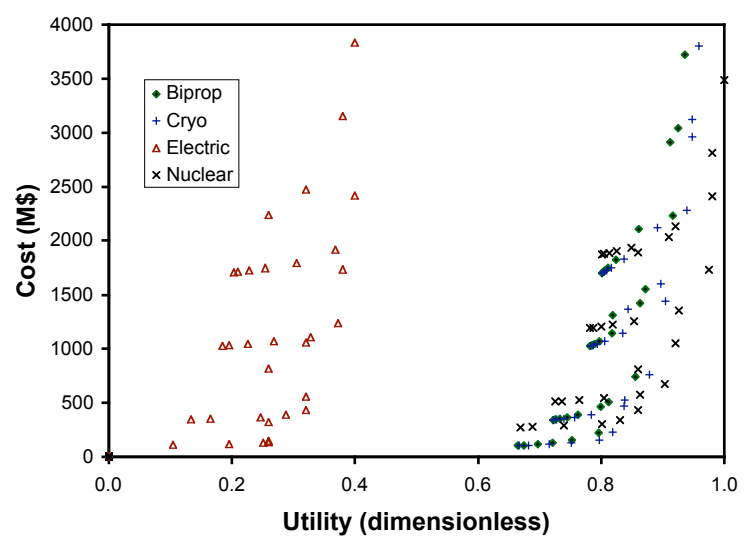

Fig. 7. Trade space for response time stressed user

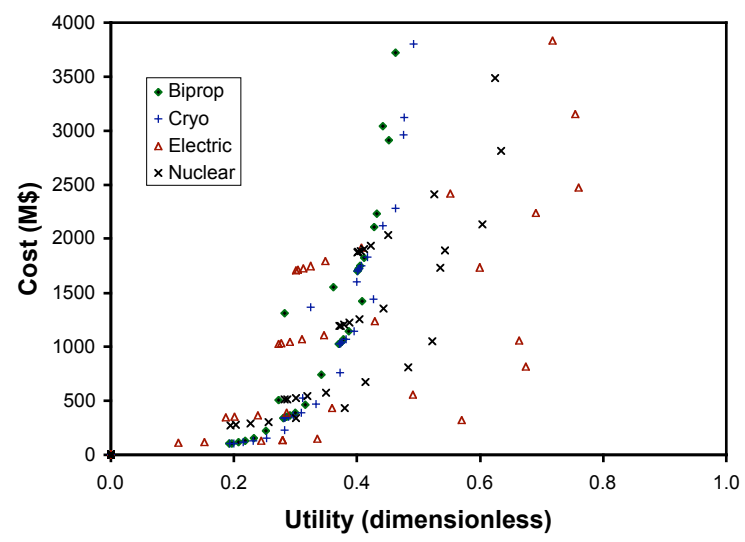

Fig. 8. Trade space for user with large delta-V needs
All the propulsion systems appear to hit a "wall" where costs increase sharply at little or no advantage in utility. Examination of the designs on this wall reveal two very different phenomena. The bi-propellant and cryogenically fueled systems are up against the limits of the rocket equation. Each small increment in utility is gained only by carrying a lot more fuel, most of which is used to push fuel around. The nuclear and electric systems, on the other hand, are limited only by the fact that they achieve a high enough delta- $\mathrm{V}$ to score a 1.0 on the delta- $\mathrm{V}$ utility, and there is simply no value in carrying more fuel. If that limit is removed, both systems show large advantages, as shown in Fig. 8.

Also shown on Fig. 9 are some specific designs capable of carrying out the mission mentioned in the introduction - moving from a LEO parking orbit to GEO transfer orbit, grappling a stranded target vehicle, inserting it in GEO, and (optionally) returning to LEO.

The biprop design is "on the wall", needing a very large fuel load to create the necessary delta-V. The cryogenically fueled design is not as bad, but is clearly sensitive to the details of its design - slight increases in manipulator mass etc. will send it too "up the wall."

Neither chemical fuels can (without refueling) return a vehicle to LEO. The electric vehicles, both one-way "tug" and round-trip "cruiser" do not have this problem. The Electric Cruiser design, in fact, sits in the lowerright corner of the tradespace because it has maximized the delta-V utility, not because it is limited by physics.

To flesh out the vehicles briefly described here, and verify the reasonableness of the very approximate methods used in the tradespace analysis, conceptual designs for these vehicles were created using ICE.

\section{ICE METHOD}

ICE is a way of streamlining the design process to make it more efficient. ICE addresses some of the major problems of spacecraft development including complicated interdisciplinary interfaces and inefficient time usage. Caltech's Laboratory for Spacecraft and Mission Design has made several important contributions to the ICE method, including the ICEMaker software that was used throughout the project.

ICEMaker is a parameter exchange tool that facilitates sharing of information amongst the design team. The design problem is broken down into individual modules, also known as "sheets"" or "clients" which are linked together via the ICEMaker server. Users can query the server to either send their latest numbers or receive any recent changes made in other modules that affect their work. The querying process is manual, preventing values from being overwritten without permission from the user. The combination of a human expert and a computational module is referred to as a "chair."

6

American Institute of Aeronautics and Astronautics 


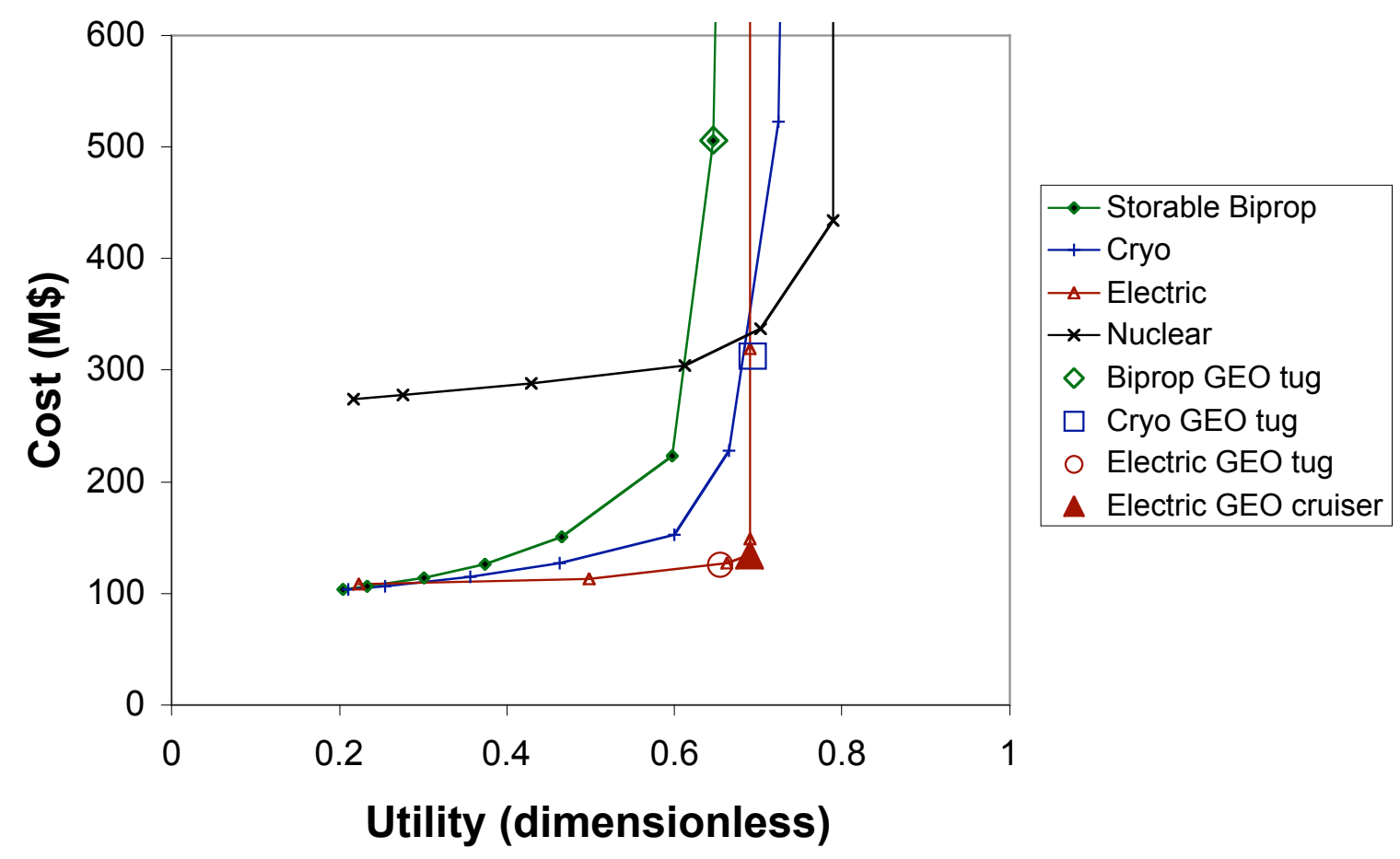

Fig. 9. Low capability systems, showing effect of increasing fuel load, with GEO rescue vehicles

ICE design sessions typically last several hours and usually address one major trade per design session. A senior team member, or "facilitator," leads the design sessions and helps to resolve disconnects between the clients. The design sessions are iterative, with each subsystem sending and receiving many times in order for the point design to converge. Although it has recently become possible to automate this iterative process, human operation of the client stations is almost always preferred. The human element is actually key to the method. The human expert can guide the iterations, catching bugs, nonsensical answers, divergence, and other pathologies that complex computational systems are prone to. More importantly, the experts make major discontinuous design decisions, or go "outside the box" by stretching parameter ranges or even adding new computational capabilities, making the ICE method a true design tool, not just a non-linear equation solver.

\section{$\underline{\text { ICE Model }}$}

Each session was broken down into three segments: pre-processing, design, and post-processing. Customer inputs, payload design, and mission objectives were decided by an Architecture chair during pre-processing. These inputs were fed to the design team and were used to develop a point design. Finally, cost was estimated during the post-processing segment.

Ten ICEMaker modules were developed, with each module representing a different spacecraft subsystem or discipline. The six main modules were Mission, Systems, Propulsion, Link, Configuration, and Power. Each sheet performed all the calculations necessary to design its specific subsystem based on the inputs provided to it. The models were developed using first principles whenever possible, but rules-of-thumb based on current technology were also used to reduce complexity and coding time. These sheets were electronically linked through the ICEMaker server and interacted throughout a design session sharing information and updating each other of changes to the design made by the individual chairs. The ICEMaker server works primarily with Microsoft Excel ${ }^{\mathbb{R}}$ spreadsheets. This work also made innovative use of a new software tool (Oculus $\mathrm{CO}^{\circledR}$ ) that was used to link routines written in Mathworks Matlab ${ }^{\circledR}$ and a parametric solid geometry model done in Solidworks ${ }^{\circledR}$ to the spreadsheets.

Several key simplifying assumptions were made. First, the sheets were only required to handle one vehicle per 
design session. The Mating and Payload subsystems were treated as "black boxes" with their specifications (mass, power, volume) fixed during the pre-processing segment by the Architecture chair. Software, control systems, and operations were not considered beyond a costing rule of thumb. Finally, a few aspects of the vehicle design were handled by "dummy chairs" at a low level of model complexity. Structures, Thermal, Attitude Control, and Command and Data Handling historically have a low impact on overall vehicle design at this level of analysis and can be handled adequately by rules of thumb. These dummy chairs can easily be expanded for future work without changing the overall architecture if desired.

The following is a summary of the six main ICEMaker modules including their inputs and outputs:

Mission: determines delta- $\mathrm{V}$ requirements and other high-level specifications

- Inputs - target orbits, tasks, timeline

- Outputs - orbital elements, mission sequence, delta-Vs, lifetime, mission duration

Propulsion: sizes the propulsion subsystem, determines

fuel requirements

- Inputs - initial dry mass, delta Vs, thrust requirements, target satellite masses, refueling requirements

- Outputs - fuel mass and volume, propulsion system type with mass and power requirements, wet mass of Space Tug

Power: sizes the power subsystem

- Inputs - power requirements (average and peak) from each subsystem by mode, orbit periods and eclipse length by phase

- Outputs - solar array mass and area, battery and power management mass, temperature constraints

Link: sizes the telecommunications subsystem, calculates mission link budget

- Inputs - transmit station location, Space Tug orbit parameters, uplink and downlink margins, total data rate, mode durations

- Outputs - antenna type and dimensions, power requirements by mode, telecomm subsystem mass

Configuration: produces a visual representation of the vehicle

- Inputs - system hardware dimensions and mass, fuel volume

- Outputs - inertia tensor, surface areas, CAD model

Systems: maintains summaries of all major

specifications (mass, power, etc.)

- Inputs - mass by subsystem, power consumption by mode, total delta $\mathrm{V}$, overall dimensions

- Outputs - total wet and dry mass by mode, link budget, cost estimate, contingencies, margins, mission summary

A summary of the ICE model and the main module interactions are illustrated in Fig. 10.

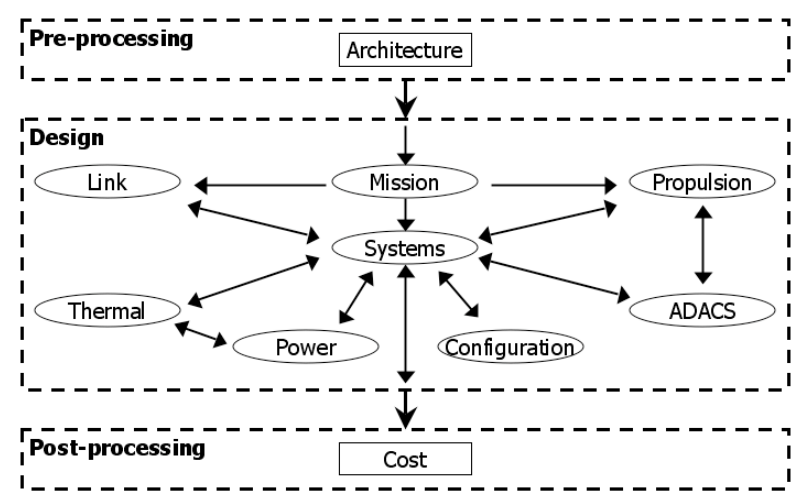

Fig. 10. ICE model components and interactions

A full MATE-CON analysis would include the tradespace analysis explicitly in the above modeling system. In this effort, the MATE model was often run concurrently with the ICE session, with key system parameters passed manually, so that the position of the developing design on the tradespace (as shown in Fig. 9) could be tracked in real time.

\section{$\underline{\text { ICE Results }}$}

Two main mission architectures were studied using the Space Tug ICE model: a GEO Tug and a GEO/LEO Tender. The GEO Tug is parked in LEO and waits for a single target mission, nominally a cooperative target of up to $2000 \mathrm{~kg}$ stranded in GEO transfer orbit. It then rendezvous with the target and inserts it into a GEO orbit, and if possible returns itself to LEO. The $\mathrm{GEO} / \mathrm{LEO}$ Tender is parked in a populated, target-rich orbit and performs multiple missions during its lifetime. Possible missions include moving or disposing of targets near its original parking orbit. Both of these architectures assume a $300 \mathrm{~kg} / 1 \mathrm{~kW}$ mating device.

\section{GEO Tugs}

Tugs were designed for both one-way and round-trip missions using three different propulsion systems: bipropellant, cryogenic, and electric. The bipropellant and cryogenic round-trip missions could not close their delta-V budgets, leaving four feasible designs. Table 5 and Figs. 11-13 summarize the GEO Tug designs. The masses and power figures are taken from the ICE session results. The delta-V, utility, and cost numbers are taken from the MATE analyses to allow direct comparison to the tradespace results (e.g. Fig. 9). The ICE system created considerably more design detail than shown in Table 5. Mass, power, and link budgets were created-see Fig. 14 for a typical result. The physical sizes and layouts of major components were also determined and linked to a parametric solid model, which can be seen in Fig. 11-13. The view in Fig. 12 shows internal layout. 
Table 5. GEO Tug Design Summary

\begin{tabular}{lcccccc}
\hline \hline Design & Dry Mass $(\mathrm{kg})$ & Wet Mass $(\mathrm{kg})$ & Power $(\mathrm{w})$ & Delta-V $(\mathrm{km} / \mathrm{s})$ & Total Utility & Cost $(\mathrm{M} \$)$ \\
\hline Biprop one-way & 1300 & 11700 & 1200 & 5.5 & 0.65 & 510 \\
Cryo one-way & 1100 & 6200 & 1200 & 7.1 & 0.69 & 310 \\
Electric one-way & 700 & 1000 & 3600 & 9.8 & 0.65 & 130 \\
Electric cruiser & 700 & 1100 & 3600 & 12.6 & 0.69 & 140 \\
\hline \hline
\end{tabular}

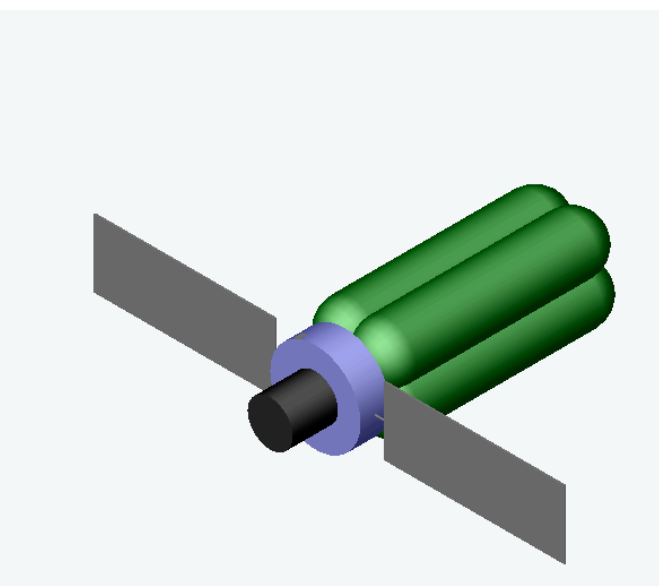

Fig. 11. Cryo one-way tug, showing extremely large fuel tanks; Bi-prop tug appears similar

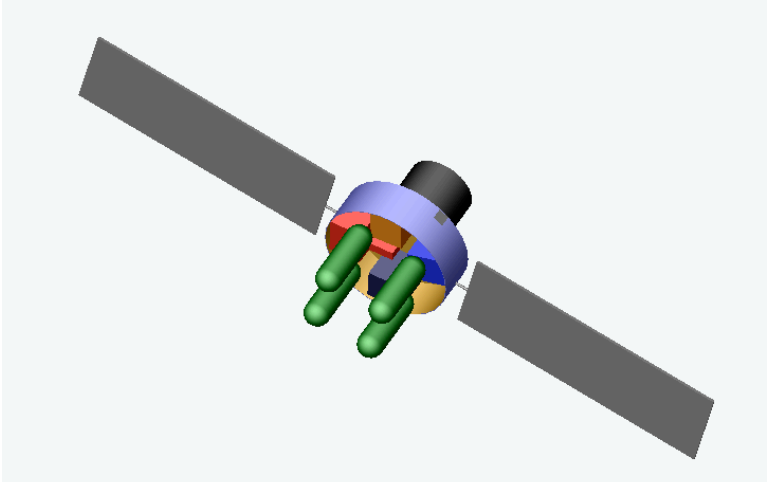

Fig. 12. Electric Cruiser (GEO round-trip tug)

The bi-prop one-way tug is very large and therefore very expensive. It is also very sensitive to changes in any of the design assumptions; any increase in dry mass causes a very large increase in fuel required. There is some danger that such a design would not "close" (i.e. the required fuel mass would become infinite) if the dry mass fraction or delta- $\mathrm{V}$ requirements were greater than anticipated. The best that can be said is that such a vehicle could fill a niche for missions where a large payload must be moved quickly using existing technology.

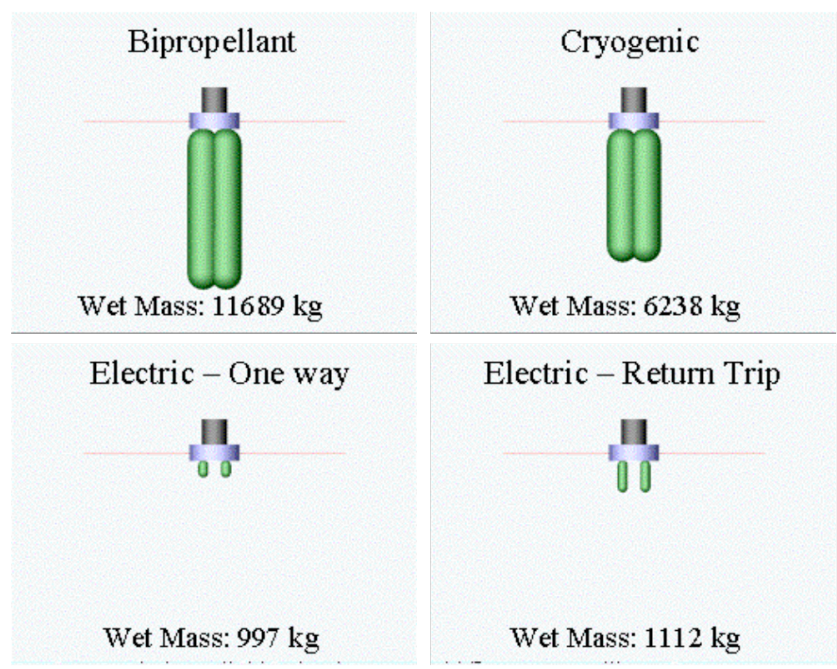

Fig. 13. Comparison of all GEO Tug designs

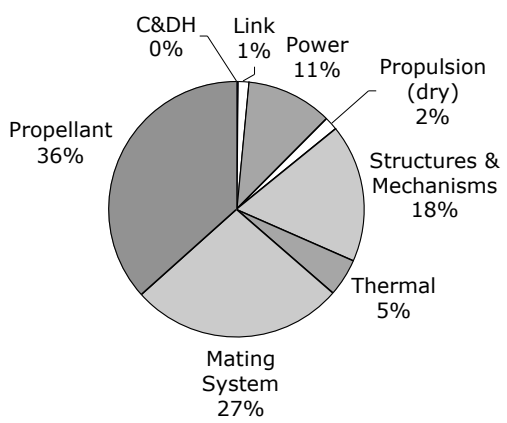

Fig. 14. Mass breakdown of Electric Cruiser design

The cryo one-way tug is significantly lighter than the biprop tug, but is almost as large due to low fuel density. It would have a very limited life on-orbit due to the need to keep the fuel cold. It is less sensitive to mass fractions and other assumptions, but still cannot make a round trip to GEO.

The electric one-way and round-trip tugs seem to be practical, versatile designs with reasonable sizes and costs. The electric designs do have the drawback of slow transit time, but they appear to be well suited for 
missions where speed is not essential. The design impact of the large total power requirement seen in Table 5 can be minimized by managing power use. Not running the manipulator and the full thruster set all at once, and trading thruster power (and hence impulse) vs. solar panel size results in panels not much bigger than those required for the chemical propulsion designs (see Figs. 11 and 12).

\section{GEO / LEO Tenders}

A family of tender missions was developed based on research of target satellite population densities. All of the tender missions use storable bipropellant systems for reduced cost and complexity. Each tender lives in a heavily populated orbit and is capable of performing five or more missions involving moving or disposing of satellites near that orbit. The result of the tender study was a line of similar vehicles with different fuel loads depending on the delta $\mathrm{V}$ requirements of the desired orbit. These designs are discussed in the companion paper.

\section{A note on model consistency and accuracy}

The differences between the results of the detailed ICE and very simple MATE analyses were remarkably small. Calculated masses differed by only a few percent. The only exceptions were the chemical fuel one-way GEO tug designs, due to their extreme fuel loads. These differences did not affect the points made here. Power was not calculated by the MATE model. Delta-V was calculated differently by the ICE and MATE models, with the ICE model taking into account the details of the mission including rendezvous maneuvers and the masses of target vehicles, but the results were consistent given this difference. A check of the ICE models' Theoretical First Unit (TFU) plus launch costs against the simple MATE cost model again showed remarkable agreement (within $15 \%$ in all cases). The ICE model also included development and engineering cost outputs, but these were not used due the wide variation in technological maturity between the different propulsion systems considered, which the model made no provision for.

The above comparison, along with sensitivity study carried out for the MATE analysis, and the relative simplicity of the calculations, help verify that the models are accurate predictors of their outputs, for the often estimated or parametric inputs used. The model results should therefore be useful for ranking and discussion, but the values given in all cases should be taken to be estimates with accuracy appropriate for concept evaluation and comparison only.

\section{DISCUSSION}

The tradespace analyses clarify the challenges of designing spacetug vehicles. Visualization of the many possible solutions to the problem of moving mass around in near-earth orbits reveals key constraints and trades, and concentrates attention on a set of viable solutions. The rapid conceptual designs help to validate the crude tradespace models, further clarify design issues, and add detail and credibility to the designs of viable vehicles. The combined set of models represents a capability that can be exercised to look at the specific needs of customers (by identifying their utilities); exploring the possibilities of specific missions (by designing vehicles for them, and understanding their position in the overall tradespace) and investigating the impact of specific technologies (by adding them to the tradespace and/or design analyses, and seeing the results).

A number of lessons that should be widely applicable to this class of vehicle were learned during the study. First, the unfriendly physics of high delta-V missions (the "rocket equation wall") make carrying out these missions with chemical propulsion systems problematic. Even if a design of this type looks feasible, it will be very vulnerable to unforeseen increases in mass fraction and/or mission requirements, and it may be very expensive. Higher specific impulse systems show promise, although caveats are in order. The electric propulsion systems examined appeared to offer the best overall mix of performance and cost, but at the expense of speed. The postulated missions are somewhat outside the current range of experience with these technologies - it was assumed, for example, that getting operating lifetimes beyond those of current systems would be feasible. Nuclear systems look interesting if there is need for very high-capability systems with quick response times; they are the only technology studied that can meet such a need. They are always expensive however, and the costs quoted here do not include any technology development. Also, the policy and/or political issues surrounding this technology were not addressed here. Methods for quantifying the costs of policy choices were recently studied by Weigel, ${ }^{11}$ and could be applied to this case.

The comparison of the performance of current and near future propulsion systems give hints as to the potential value of other technologies applied to this problem. A high- $I_{s p}$, high impulse system without the large mass penalty of a nuclear system would be ideal; solar thermal, or stored-solar-energy systems (e.g. flywheel storage) might be worth investigating for this purpose. On the other hand, the good results with existing electric propulsion options make other low thrust (e.g. solar sail) technologies less interesting, unless there is a very large demand for delta- $\mathrm{V}$. The trade-off between $I_{s p}$, impulse, and total delta-V was found to be very sensitive to user needs. Thus, any further discussion of the value of various propulsion systems needs to take place in the context of the needs of a real user or at least a more completely specified desired capability.

An issue that was relatively insensitive to user needs was the high penalty for dry mass on the tug vehicle. The higher capability (higher observation and manipulator mass) vehicles showed large cost penalties. 
Put another way, the total system costs were highly sensitive to the efficiency of the observation and manipulation systems. Any user would be motivated to achieve the highest actual capability for the lowest mass (and, secondarily, power) when designing such equipment.

The current tradespace analysis reveals three classes of potentially useful space tug vehicles. They are highlighted on Fig. 15. The Electric Cruiser occupies the "knee in the curve" for our nominal utilities, providing good value for cost. It could potentially provide even more value for a delta-V hungry user (see Fig. 8) although it is sensitive to user needs for response time. Its features have been discussed in this paper. The "Nuclear Monsters" were not discussed here, but appear to be the only designs (out of the design space considered) that can provide high delta-V, high capability, rapid response systems. A final range of vehicles occupies the lower left region of the Pareto front. These are cost effective vehicles built using existing technology (e.g. storable bi-propellant systems) that can do a variety of jobs requiring less delta- $V$ than a LEO-GEO transfer. They could, for example, tend sets of vehicles in similar orbits, doing a variety of maintenance tasks. For this reason (and to extend the naval support vessel metaphor) we have dubbed them "Tenders." They are considered in depth in the companion paper.

\section{ACKNOWLEDGEMENTS}

This work was performed by the authors, MIT students Laura Condon, Todd Wesley, Devjit Chakravarti, Gerganna Bounova, Lisa Messeri, and Matt Richards, and Cambridge University students Nishant Lalwani and Bill Cunliffe. The work was sponsored by DARPA (TTO) with Dr. Gordon Roesler as Program Manager. Funding was administerd via AFRL and the "Grand Challenges" contract number F29601-97-K-0010. Ms. Charlotte Gerhart served as the AFRL project manager.

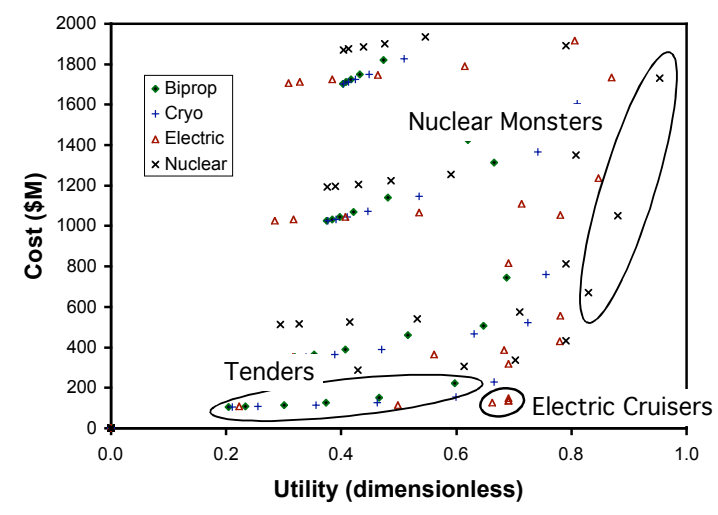

Fig. 15. Promising designs

\section{REFERENCES}

1. Galabonva, K., Bounova, G., de Weck, O. and Hastings, D., "Architecting a Family of Space Tugs Based on Orbital transfer Mission Scenarios," AIAA paper 2003-6368.

2. Saleh, J. H., Lamassoure, E., and Hastings, D. E. "Space Systems Flexibility Provided by On-Orbit Servicing: Part 1," Journal of Spacecraft and Rockets, Vol. 39, No. 4, July-Aug. 2002, pp. 551560.

3. McManus, H. L., and Warmkessel, J. M., "Creating Advanced Architectures for Space Systems: Emergent Lessons from New Processes," Journal of Spacecraft and Rockets, in press, modified from AIAA paper 2001-4738.

4. Shaw, G. M., Miller, D. W., and Hastings, D. E., "Development of the Quantitative Generalized Information Network Analysis (GINA) Methodology for Satellite Systems," Journal of Spacecraft and Rockets, Vol. 38, No. 2, 2001, pp. 257-269.

5. Smith, J. L., "Concurrent Engineering in the JPL Project Design Center," Society of Automotive Engineers, Inc., Paper 98AMTC-83, 1998.

6. Aguilar, J. A., and Dawdy, A., "Scope vs. Detail: The Teams of the Concept Design Center," 2000 IEEE Aerospace Conference Proceedings, Big Sky, Montana, March 2000, Vol. 1, pp. 465-482.

7. Parkin, K., Sercel, J., Liu, M., and Thunnissen, D., "ICEMaker: An Excel-Based Environment for Collaborative Design," 2003 IEEE Aerospace Conference Proceedings, Big Sky, Montana, March 2003.

8. McManus, H. L., Hastings, D. E., and Warmkessel, J. M., "New Methods for Rapid Architecture Selection and Conceptual Design," Journal of Spacecraft and Rockets, in press.

9. Ross, A. M., Diller, N. P., Hastings, D. E., and Warmkessel, J. M., "Multi-Attribute Tradespace Exploration as a Front-End for Effective Space System Design," Journal of Spacecraft and Rockets, in press.

10. "Project Freebird: An Orbital Transfer Vehicle", Final report, 16.83 Space Systems Engineering, Aeronautics and Astronautics Department, MIT, Spring 1994.

11. Weigel, A. L., and Hastings, D. E., "Evaluating the Cost and Risk Impacts of Launch Choices," Journal of Spacecraft and Rockets, in press.

11

American Institute of Aeronautics and Astronautics 